\title{
Effects of intraruminal propionate supplementation on nitrogen utilisation by the portal-drained viscera, the liver and the hindlimb in lambs fed frozen rye grass
}

\author{
Isabelle C. Savary-Auzeloux ${ }^{1 *}$, Linda Majdoub ${ }^{1}$, Nathalie LeFloc'h ${ }^{2}$ and \\ Isabelle Ortigues-Marty ${ }^{1}$ \\ ${ }^{1}$ Unité de Recherche sur les Herbivores, Equipe Nutriments et Métabolismes, INRA Clermont Ferrand-Theix, \\ 63122 St Genès Champanelle, France \\ ${ }^{2}$ Unité Mixte de Recherche sur le Veau et le Porc, INRA-ENSAR St Gilles, 35590 St Gilles, France
}

(Received 17 September 2002 - Revised 3 July 2003 - Accepted 7 July 2003)

\begin{abstract}
The influence of propionate supplementation on the splanchnic metabolism of amino acids (AA) and other $\mathrm{N}$ compounds (urea- $\mathrm{N}$ and $\mathrm{NH}_{3}-\mathrm{N}$ ) and the supply of AA and $\mathrm{NH}_{3}-\mathrm{N}$ to the hindlimb was investigated in growing lambs. Six rumen-cannulated and multicatheterised lambs $(32.2 \mathrm{~kg})$ were fed frozen rye grass at $690 \mathrm{~kJ}$ metabolisable energy intake/d per kg average metabolic body weight. They were infused intraruminally with a salt solution (control) or with propionate solutions at $0.23 \mathrm{~mol} / \mathrm{l}(\mathrm{P} 1)$ or $0.41 \mathrm{~mol} / \mathrm{l}(\mathrm{P} 2)$ infused at a maximal rate of 1.68 (SD 0.057) $\mathrm{ml} / \mathrm{min}$ according to a repeated Latin square design. The propionate infusion did not increase the net portal appearance of total AA (TAA)-N but increased that of some branched-chain AA (valine and to a lesser extent isoleucine). Simultaneously, the propionate treatment (especially P2) induced an increased TAA utilisation by the liver. This was due mainly to an increased (+79\%; $P<0.07)$ utilisation of the essential AA and particularly the branched-chain AA. A stimulation of protein synthesis in the liver is hypothesised since (1) propionate stimulated insulin secretion and (2) utilisation of non-essential AA were less influenced by the propionate treatment in the liver (except for alanine), suggesting that the AA utilised by the liver were directed towards protein synthesis rather than towards oxidation or urea synthesis. At the splanchnic level, the propionate treatment did not have any effect on the TAA, non-essential AA and essential AA, except for a net splanchnic release that was decreased for leucine $(P<0.02)$ and methionine $(P<0.01)$ and increased for threonine $(P<0.05)$. The propionate treatment did not have any effect on the hindlimb uptake of AA (essential and non-essential). As a consequence, even though the propionate treatment induced some major alterations in the splanchnic metabolism of AA, there were no changes in the net AA balance in the hindlimb (and hence probably on muscle growth). The role of the splanchnic tissues in the regulation of the AA supply to the peripheral tissues (such as muscle) therefore appears to be prominent in the regulation of muscle growth. Whether the peripheral tissues regulate their own supply by interacting with the splanchnic tissues (and especially the liver) or the liver is the only regulator of the AA supply to the muscle remains in doubt.
\end{abstract}

Propionate: Splanchnic tissues: Hindlimb: Amino acids: Net flux

Growing ruminants fed on fresh grass or grass silage are characterised by an insufficient supply of energy and digestible protein in the gut to satisfy high growth requirements (Institut National de Recherches Agronomiques, 1978). To improve the metabolisable energy (ME), $\mathrm{N}$ intake and growth of animals fed fresh forages, a concentrate supplementation is often applied. However, when such a supplementation is applied, energy and $\mathrm{N}$ supply are increased simultaneously. Consequently, it is hard to distinguish the direct effect of $\mathrm{N}$ supplementation from the indirect effect of energy supplementation (via sparing processes of amino acids (AA) at the portal-drained viscera (PDV), liver or peripheral tissues levels) on $\mathrm{N}$ metabolism in the different tissues and organs.

An increase in propionate absorption from the rumen has been previously shown to increase milk and milk protein yield (Huhtanen et al. 1998), carcass protein deposition (Hovell \& Greenhalgh, 1978; Moloney, 1998) and protein synthesis (Abdul-Razzaq \& Bickerstaffe, 1989) in the muscle of ruminants. An increase in propionate absorption can be brought about by a direct intraruminal infusion of propionate or after a diet manipulated in favour of a propionic fermentation profile. However, conflicting results were found by Harmon \& Avery (1987), who found a

Abbreviations: AA, amino acids; BCAA, branched-chain amino acids; EAA, essential amino acids; Glx, glutamate + glutamine; ME, metabolisable energy; MET, metabolites; NEAA, non-essential amino acids; NPA, net portal appearance; P1, 0.23 M-propionate infusion; P2, 0.41 M-propionate infusion; PDV, portal-drained viscera; PFp, portal plasma flow; TAA, total amino acids; VFA, volatile fatty acids.

* Corresponding author: Dr Isabelle Savary-Auzeloux, fax +33 4736246 39, email Savary@clermont.inra.fr 
decrease in net portal flux of $\alpha$-amino-N after the addition of sodium propionate in concentrate-fed steers. Similarly, Hovell \& Greenhalgh (1978) did not find any effect of propionate salts $(22 \%$ of the total ME intake) on lamb growth. Most of the studies in growing ruminants or nonruminants supplemented with volatile fatty acids (VFA) have examined (1) the net portal appearance (NPA) of the $\mathrm{N}$ compounds using arterio-venous differences (Seal et al. 1993; Nozière et al. 2000), (2) the utilisation of the $\mathrm{N}$ compounds by the liver, particularly the effect of propionate on ureagenesis (in vitro results; Demigné et al. 1991), (3) $\mathrm{N}$ balance and the carcass composition (Moloney, 1998), or (4) protein synthesis and AA balance in the muscle (Abdul-Razzaq \& Bickerstaffe, 1989). No study so far has examined these aspects together to obtain an integrated view of the effects of VFA supplementation on $\mathrm{N}$ utilisation by the tissues and organs that are the major $\mathrm{N}$ consumers in growing, meat-producing ruminants; the PDV, the liver and the muscle. Moreover, no data are available on the net fluxes of $\mathrm{N}$ nutrients in growing ruminants fed grass.

The PDV and the liver are two major sites of utilisation of nitrogenous nutrients. Indeed, they represent up to $30-40 \%$ of the whole-body protein synthesis (Lobley, 1994) and up to $50 \%$ of the overall energy expenditure (Ortigues \& Doreau, 1995; Majdoub et al. 2003). These two organs, because of their very active metabolism and their anatomical position, influence the amount and the pattern of AA supplied to the muscle (Lobley, 1994). In a situation of limited supply of digestible protein in the gut (in addition to a limited supply of energy), the use of AA to cover the energy requirements of splanchnic tissues (Lapierre \& Lobley, 2001) would further penalise the limited NPA of AA. In addition to the normal utilisation of AA for protein synthesis in the liver, the hepatic detoxification of large amounts of $\mathrm{NH}_{3}$ to urea (Milano et al. 2000) could also create another drain on AA and further limit AA availability to the peripheral tissues. In the liver, the AA can also be used for gluconeogenesis, particularly when glucose precursors (especially propionate) are insufficient (Kelly et al. 1993; Van der Walt, 1993). The negative consequences of splanchnic N metabolism on AA availability to peripheral tissues might be limited by an increased availability of energy-yielding substrates. These might act by sparing the AA (Seal \& Parker, 1996), which could then be used for protein synthesis instead of for energetic purposes in the PDV and the liver (Seal et al. 1993). This mechanism could then improve AA availability to the peripheral tissues such as muscle (Lapierre et al. 2000).

The present study is part of a larger research programme on meat quality at grass. Thus the aim of the present study was to investigate the effect of an exogenous propionate supply in growing ruminants fed frozen rye grass on the PDV release of $\mathrm{N}$, the utilisation and net release of $\mathrm{N}$ by the liver and the utilisation of the AA by the peripheral tissues (and especially the muscle). This integrated view could provide information on where (at the PDV or the liver level) and how (via some sparing mechanism of the AA or via hormonal regulation involving insulin, for instance) the energy supply can help to improve muscle growth. A companion paper has already shown that propionate infusion alters the fluxes of energy compounds and insulin (Majdoub et al. 2003).

\section{Materials and methods}

\section{Animals, diet and treatments}

Six INRA 401 lambs of 31 (SD 4.0) $\mathrm{kg}$ were surgically fitted with a rumen cannula (12 $\mathrm{mm}$ internal diameter) and chronic blood catheters in the portal vein, a hepatic vein, an external iliac vein and a mesenteric artery. Splanchnic catheters were introduced as described by Ortigues \& Durand (1995). The external iliac catheter was introduced via the femoral vein and the tip was positioned just between the two bifurcations of the deep femoral vein and circumflex iliac vein (Majdoub et al. 2003). Lambs were also fitted with two ultrasonic blood-flow probes (Transonic Systems Inc., Ithaca, NY, USA) around the portal vein (16A) and an external iliac artery (3R) and the blood flows were recorded using a flowmeter (T206; Transonic Systems Inc.). Animals were housed in individual stalls with ad libitum access to drinking water and salt lick, under continuous lighting. After a recovery period from surgery of a minimum of $8 \mathrm{~d}$, lambs were adapted to the basal diet during 2 weeks. Lambs received perennial rye grass (first cut, fertilised at $80 \mathrm{~kg} \mathrm{~N} / \mathrm{ha}$ ), harvested at grazing stage, chopped in $50 \mathrm{~mm}$ lengths, frozen at $-35^{\circ} \mathrm{C}$ and stored at $-15^{\circ} \mathrm{C}$ at an estimated level of $690 \mathrm{~kJ} \mathrm{ME} / \mathrm{kg}$ average metabolic body weight as twelve equal meals. Since the quality of fresh grass varies temporally, the rye grass was frozen in order to obtain a homogeneous batch of grass whose properties (in terms of degradability and digestibility) were as close as possible to fresh grass. Rye-grass ME content was estimated at $11.6 \mathrm{MJ} / \mathrm{kg} \mathrm{DM}$ (Institut National de Recherches Agronomiques, 1978). The contents of ryegrass-N, crude fibre and soluble sugars were 3.35 (SD 0.06 ), 21.22 (SD 0.55) and 10.89 (SD 0.85) \% on a DM basis, respectively (Majdoub et al. 2003). The rye-grass DM content measured on a daily basis averaged 19.6 (SD 2.58) \%. The content of soluble $\mathrm{N}$ was $25.6 \%$ of total N. Lambs received three treatments according to a replicated $3 \times 3$ Latin square design at an average experimental weight of 32 (SD 2.2) kg. Treatments consisted of $8 \mathrm{~d}$ of continuous intraruminal infusion of a salt solution ( $\mathrm{Na}$ and $\mathrm{K}$ ) for the control treatment, or of a propionate solution at $0.23 \mathrm{M}$ for the propionate 1 (P1) and at $0.41 \mathrm{M}$ for the propionate $2(\mathrm{P} 2)$ treatments as described previously (Majdoub et al. 2003). All solutions were buffered to $\mathrm{pH} 4 \cdot 6$, with $\mathrm{HCl}$ for the control treatment and with $\mathrm{NaOH}(3 \mathrm{M})$ and $\mathrm{KOH}(1 \mathrm{M})$ for $\mathrm{P} 1$ and $\mathrm{P}$ 2, and supplied the same daily amount of $\mathrm{Na}$ and $\mathrm{K}(4.97 \mathrm{Na} / \mathrm{d}$ and $2.82 \mathrm{~K} / \mathrm{d}$ ). Solutions were infused at a maximal rate of 1.68 (SD 0.057) $\mathrm{ml} / \mathrm{min}$. The propionate infusions supplied 69 and $110 \mathrm{~kJ} \mathrm{ME} / \mathrm{kg}$ average metabolic body weight and represented 10 and $16 \%$ of the daily ME allowances from rye grass (for P1 and P2 respectively). The treatment periods were separated by a transition period of $7 \mathrm{~d}$. The infusions had no effect on rumen $\mathrm{pH}$ and osmolarity. 
The experiment was conducted in a manner compatible with national legislation on animal care (Certificate of Authorisation to Experiment on Living Animals, no. 004495, Ministry of Agriculture).

\section{Sampling procedures and laboratory analyses}

On the last day of infusion, blood flows in the portal vein and in the external iliac artery were continuously recorded over $4 \mathrm{~h}$ (over two feeding cycles, between 11.00 and 15.00 hours). Hepatic arterial blood flow was estimated at $5.3 \%$ of the portal blood flow based on the reported contribution to hepatic blood flow (Barnes et al. 1986; Milano et al. 2000). Some of the net balances of metabolites (total AA (TAA), non-essential AA (NEAA), essential AA (EAA), branched-chain AA (BCAA), urea-N and $\mathrm{NH}_{3}-\mathrm{N}$ ) have been recalculated using a contribution of the hepatic arterial blood flow of $15 \%$ of the portal flow (data not shown). In the present study, the influence of this contribution in the calculation of the net hepatic and total splanchnic tissue net fluxes is very low and the interpretation of the data in terms of 'propionate effect' remains the same. Consequently, the hepatic and total splanchnic tissue net fluxes have been calculated using a contribution of the arterial blood flow of $5.3 \%$ of the portal flow. To facilitate the interpretation of hindlimb blood flows (which fluctuate depending on the position of the animal), visual observations were made of the behaviour of the animals during this period and blood sampling was performed only in quietly standing animals. Eight sets of blood samples were taken from the portal vein, the hepatic vein, the external iliac vein and from the mesenteric artery every $30 \mathrm{~min}$ and starting $15 \mathrm{~min}$ postprandially. Blood $(2.5 \mathrm{ml})$ was taken in tubes containing $3 \mathrm{~mm}-$ EDTA-K (10 $\mu \mathrm{l} / \mathrm{ml}$ blood) (Sigma, St Louis, MO, USA) as anticoagulant and centrifuged at $4^{\circ} \mathrm{C}$. The packed cell volume was determined on the blood before centrifugation. Plasma was frozen at $-20^{\circ} \mathrm{C}$ for the later analysis of $\mathrm{NH}_{3}$ (Bergmeyer (1985) according to the Berthelot method), urea (KitS-1000; bioMérieux, Marcy l'Etoile, France) and AA. The storage at $-20^{\circ} \mathrm{C}$ of the plasma samples for AA analysis induced a partial degradation of glutamine into glutamate and asparagine into aspartate. Consequently, the data for glutamate and glutamine (Glx) were combined as well as the data for aspartate and asparagine.

The AA concentrations were determined on pooled plasma samples after deproteinisation of $0.65 \mathrm{ml}$ plasma with $0.24 \mathrm{M}$-sulfosalicylic acid $(0.65 \mathrm{ml})$ by ion-exchange chromatography (Biotronik LC 5001; Biotronik, Pusheim Banhof, Germany), with ninhydrin as the colorimetric reagent.

Three samples of rumen fluid $(20 \mathrm{ml})$ were taken every $30 \mathrm{~min}$ over one feeding cycle (between 15.00 and 17.00 hours). The analyses performed on the rumen fluid samples are detailed in the companion paper (Majdoub et al. 2003).

\section{Calculations and statistical analyses}

Net nutrient fluxes through the PDV, the liver and the hindlimb were calculated as described by Katz \& Bergman (1969). The net nutrient fluxes were calculated as differences between the afferent flux and the efferent flux. Consequently, a positive net flux indicates a net release whereas a negative net flux indicates a net uptake.

The NPA of metabolites (MET) was calculated as follows:

$$
\begin{aligned}
\mathrm{NPA}= & \left(\text { concentration of } \mathrm{MET}_{\mathrm{PV}}\right. \\
& \left.- \text { concentration of } \mathrm{MET}_{\mathrm{A}}\right) \times \mathrm{PFp}
\end{aligned}
$$

where PFp is the portal plasma flow, and $\mathrm{MET}_{\mathrm{PV}}$ and $\mathrm{MET}_{\mathrm{A}}$ are the metabolites in the portal vein and the artery respectively. Since the AA were assayed in the plasma, the blood flows were transformed into plasma flows using the packed cell volume. The net hepatic flux of $\mathrm{AA}, \mathrm{NH}_{3}$ and urea was calculated as follows:

Net hepatic flux $=\left(\right.$ concentration of $\mathrm{MET}_{\mathrm{HV}} \times 1.053$

$$
\begin{aligned}
& -\left(\text { concentration of } \mathrm{MET}_{\mathrm{PV}}\right. \\
& \left.\left.+ \text { concentration of } \mathrm{MET}_{\mathrm{A}} \times 0 \cdot 053\right)\right) \\
& \times \mathrm{PFp},
\end{aligned}
$$

where $\mathrm{MET}_{\mathrm{HV}}$ is the metabolites in the hepatic vein. Last, the net flux of $\mathrm{AA}, \mathrm{NH}_{3}$ and urea across the hindlimb was calculated as follows:

Net flux across the hindlimb

$$
\begin{aligned}
= & \left(\text { concentration of } \mathrm{MET}_{\mathrm{I}}-\text { concentration of } \mathrm{MET}_{\mathrm{A}}\right) \\
& \times \mathrm{PF}_{\mathrm{I}},
\end{aligned}
$$

where $\mathrm{PF}_{\mathrm{I}}$ is the iliac plasma flow and $\mathrm{MET}_{\mathrm{I}}$ the metabolites in the iliac vein. The fractional extraction was calculated as the net flux:total entry flow ratio to the tissue. EAA included histidine, isoleucine, leucine, lysine, methionine, phenylalanine, threonine and valine; NEAA included alanine, arginine, asparagine + aspartic acid, glutamic acid + glutamine, glycine, proline, serine and tyrosine; BCAA included isoleucine, leucine and valine. TAA were the sum of EAA and NEAA.

For the splanchnic tissues, the data were analysed according to a replicated Latin square design with treatment, square, animal (square) and period as the main factors, despite the fact that measurements could not all be carried out on the same days. However, because of problems with catheter patency, the results for the hindlimb are based on four animals only and the data were analysed by ANOVA using treatment and animal as the main factors. Analyses were carried out using the general linear model procedure of Statistica version 5.5 (1984-2000; StatSoft, Tulsa, OK, USA). Means were compared by orthogonal contrasts (contrast 1: control v. (P1+P2), and contrast 2: $\mathrm{P} 1 v . \mathrm{P} 2)$ to test responses to the presence of propionate and to the level of propionate. Differences were considered significant if $P<0.05$ and as a tendency for $0 \cdot 05<P<0 \cdot 1$. 


\section{Results}

\section{Intake and ruminal fermentations}

The experiment began a minimum of 3 weeks after surgery. The $\mathrm{DM}$ as well as the $\mathrm{N}$ intake decreased by approximately $10-15 \%$ between the control and propionate-infused animals $(913,823$ and $811 \mathrm{~g}$ DM intake/d and $31.6,27.7$ and $26.2 \mathrm{~g} \mathrm{~N}$ intake/d for the control, P1 and $\mathrm{P} 2$ animals respectively; $P=0.05)$. The drop in intake measured with the P1 and P2 treatments was entirely due to a high daily variability in the DM content of frozen grass (Majdoub et al. 2003) and not to the propionate treatment. The fermentation parameters were detailed in a companion paper (Majdoub et al. 2003). Briefly, the amounts of propionate infused were 0.55 (SD 0.027) and 0.98 (SD $0.033) \mathrm{mol} / \mathrm{d}$, which induced increases of 36 and $71 \%$ in propionate concentrations in the rumen fluid for P1 and $\mathrm{P} 2$ respectively $(P<0 \cdot 001)$. Acetate and butyrate concentrations in the rumen remained stable whereas the caproate concentrations tended to decrease by $51 \%(P<0.07)$ over all the treatments. Consequently, the molar proportions of acetate in the rumen dropped from 59.2 to 53.4 and $51.3 \%(P<0.001)$ while those of propionate rose from 24.8 to 33.0 and $37.5 \%(P<0.001)$ in the control, P1 and P2 animals respectively (Majdoub et al. 2003). Rumen fluid $\mathrm{pH}$ and $\mathrm{NH}_{3}$ concentrations were not altered by the propionate infusion $(6.38$ and $10.49 \mathrm{~mm}$ on average respectively).

\section{Blood flows and amino acids concentrations}

Blood flows in the portal vein and the external iliac artery (Table 1) averaged 128.4 and $8.4 \mathrm{ml} / \mathrm{min}$ per $\mathrm{kg}^{0.75}$ respectively and were not altered by the propionate infusion. Packed cell volumes averaged $0 \cdot 27$ and were not modified by the treatments. Packed cell volumes remained stable throughout the sampling period (data not shown); consequently, the plasma flows were not altered by the propionate infusion (Table 1).
The TAA concentrations in the artery were not altered by the propionate infusion (Table 1); similarly the arterial concentrations of NEAA and EAA. Considering the EAA individually, a decrease in arterial concentrations with the propionate infusion was noted for the BCAA $(P<0.04)$ (isoleucine $(P<0.03)$ and valine $(P<0.03))$ and threonine $(P<0.05)$ by an average of $17 \%$ (Tables 1 and 2). Consequently, the relative arterial concentrations of BCAA to the TAA decreased $(P<0.04)$ after the propionate infusion $(20 \cdot 2,18.7$ and $16.8 \%$ for the control, P1 and P2 animals respectively). When considering other blood vessels, treatment effects appeared as statistically significant or as trends when arterial concentrations were significantly modified by treatments (for example, for BCAA and some NEAA such as serine and glycine; data not shown). The plasma $\mathrm{NH}_{3}-\mathrm{N}$ and the urea- $\mathrm{N}$ concentrations in the artery (as well as in the other vessels) were not altered by the propionate treatments (data not shown).

\section{Net fluxes of amino acids in the portal-drained viscera}

Control animals. The net release of $\mathrm{N}$ as TAA-N and $\mathrm{NH}_{3}-\mathrm{N}$ in the portal vein represented 15.7 and $13.5 \%$ respectively of total $\mathrm{N}$ intake and the NPA of $\mathrm{N}$ as EAA$\mathrm{N}$, NEAA-N and BCAA-N amounted to 55, 45 and $17 \%$ respectively of that of TAA-N (calculated from Table 3). On the other hand, a net transfer of urea-N was noted (22.9\% daily $\mathrm{N}$ intake) from arterial blood to the lumen of the gut (data not shown). On an individual basis (Tables 4 and 5), all the AA showed either a net positive release in the portal vein (all EAA) or a net flux, which was not $(P<0 \cdot 04)$ different from zero (mostly NEAA). However Glx were removed in substantial proportions by the PDV (-1.08 (SEM 0.24) $\mathrm{mmol} / \mathrm{h}$ for Glx in comparison with a NPA of TAA of +13.45 (SEM 1.66) $\mathrm{mmol} / \mathrm{h})$.

Propionate effects. The infusion of propionate did not significantly modify the NPA of TAA or even EAA, BCAA and NEAA (Table 3). Taking the EAA individually, the NPA of valine $(P<0.04)$ and to a lesser extent isoleucine

Table 1. Blood and plasma flows in the portal vein and external iliac artery and amino acid concentrations in the mesenteric artery in lambs fed frozen rye grass with intraruminal infusions of propionate*

(Mean values and standard errors of the mean)

\begin{tabular}{|c|c|c|c|c|c|c|c|c|}
\hline & \multirow[b]{2}{*}{$n$} & \multicolumn{3}{|c|}{ Treatments } & \multirow[b]{2}{*}{ SEM } & \multirow[b]{2}{*}{ Treatment effect } & \multicolumn{2}{|c|}{ Orthogonal contrasts $†$} \\
\hline & & C & P1 & P2 & & & Propionate & Level \\
\hline Live weight $(\mathrm{kg})$ & 6 & $32 \cdot 1$ & $32 \cdot 5$ & $32 \cdot 3$ & 0.51 & 0.59 & 0.38 & 0.62 \\
\hline $\begin{array}{l}\text { Blood liow (IItres/n) } \\
\text { Portal vein }\end{array}$ & 6 & $105 \cdot 5$ & 101.0 & $106 \cdot 0$ & $3 \cdot 13$ & 0.65 & 0.69 & 0.41 \\
\hline External iliac artery & 4 & $6 \cdot 9$ & 6.9 & $6 \cdot 8$ & 0.24 & 0.80 & 0.56 & 0.83 \\
\hline Plasma flow (litres/h) & & & & & & & & \\
\hline Portal vein & 6 & $76 \cdot 7$ & $74 \cdot 2$ & $77 \cdot 9$ & $2 \cdot 65$ & 0.71 & 0.86 & 0.43 \\
\hline External iliac artery & 4 & $5 \cdot 0$ & $5 \cdot 0$ & $4 \cdot 8$ & 0.18 & 0.88 & 0.65 & 0.88 \\
\hline TAA $(\mu \mathrm{M})$ & 6 & 2909 & 2629 & 2761 & 85.5 & 0.41 & 0.25 & 0.52 \\
\hline EAA $(\mu \mathrm{M})$ & 6 & 1090 & 928 & 919 & $41 \cdot 0$ & 0.13 & 0.05 & 0.91 \\
\hline NEAA $(\mu \mathrm{M})$ & 6 & 1819 & 1701 & 1842 & $58 \cdot 3$ & 0.54 & 0.69 & 0.31 \\
\hline BCAA ( $(\mu \mathrm{M})$ & 6 & 588 & 491 & 463 & $24 \cdot 8$ & 0.04 & 0.01 & 0.52 \\
\hline
\end{tabular}

C, control treatment; P1, 0.23 M-propionate infusion; P2, 0.41 M-propionate infusion; TAA, total amino acids; EAA, essential amino acids; NEAA, nonessential amino acids; BCAA, branched-chain amino acids.

${ }^{*}$ For details of diets and procedures, see p. 940.

†Contrast 1: C v. (P1+P2), contrast 2: P1 v. P2 
Table 2. Amino acid concentrations $(\mu \mathrm{M})$ in the mesenteric artery of lambs fed frozen rye grass with intraruminal infusions of propionate*

(Mean values and standard errors of the mean)

\begin{tabular}{|c|c|c|c|c|c|c|c|c|}
\hline & \multirow[b]{2}{*}{$n$} & \multicolumn{3}{|c|}{ Treatments } & \multirow[b]{2}{*}{ SEM } & \multirow[b]{2}{*}{ Treatment effect } & \multicolumn{2}{|c|}{ Orthogonal contrasts } \\
\hline & & $\mathrm{C}$ & $\mathrm{P} 1$ & P2 & & & Propionate & Level \\
\hline \multicolumn{9}{|l|}{ EAA } \\
\hline Histidine & 6 & $46 \cdot 0$ & $44 \cdot 0$ & $42 \cdot 7$ & 1.98 & 0.72 & 0.47 & 0.76 \\
\hline Isoleucine & 6 & $134 \cdot 7$ & $115 \cdot 7$ & $104 \cdot 0$ & 5.50 & 0.03 & 0.02 & 0.25 \\
\hline Leucine & 6 & 161.4 & $146 \cdot 1$ & 133.4 & $6 \cdot 16$ & 0.11 & 0.06 & 0.30 \\
\hline Lysine & 6 & $161 \cdot 8$ & 148.6 & $139 \cdot 8$ & $7 \cdot 27$ & 0.50 & 0.29 & 0.64 \\
\hline Methionine & 6 & $26 \cdot 2$ & $25 \cdot 9$ & $25 \cdot 5$ & 0.92 & 0.96 & 0.82 & 0.87 \\
\hline Phenylalanine & 6 & $78 \cdot 6$ & 81.4 & 74.4 & 3.62 & 0.72 & 0.93 & 0.43 \\
\hline Threonine & 6 & $189 \cdot 4$ & 137.9 & 174.0 & $13 \cdot 34$ & 0.05 & 0.06 & 0.08 \\
\hline Valine & 6 & $292 \cdot 1$ & $228 \cdot 7$ & $225 \cdot 15$ & $13 \cdot 93$ & 0.03 & 0.01 & 0.88 \\
\hline \multicolumn{9}{|l|}{ NEAA } \\
\hline Alanine & 6 & 231.5 & 226.5 & 255.5 & 9.69 & 0.45 & 0.65 & 0.25 \\
\hline Aminobutyric acid & 6 & 4.5 & 3.7 & 3.6 & 0.45 & 0.69 & 0.41 & 0.92 \\
\hline Arginine & 6 & $226 \cdot 1$ & 224.5 & $189 \cdot 6$ & $13 \cdot 77$ & 0.38 & 0.45 & 0.24 \\
\hline Aspartate + asparagine & 6 & $78 \cdot 0$ & $66 \cdot 8$ & $69 \cdot 3$ & 3.87 & 0.39 & 0.19 & 0.76 \\
\hline Citrulline & 6 & $217 \cdot 8$ & $186 \cdot 8$ & $179 \cdot 9$ & $14 \cdot 38$ & 0.09 & 0.04 & 0.68 \\
\hline Cystathionine & 6 & $1 \cdot 1$ & $1 \cdot 8$ & $1 \cdot 7$ & 0.19 & 0.33 & 0.15 & 0.85 \\
\hline Glycine & 6 & 497.5 & 461.5 & 529.9 & $27 \cdot 50$ & 0.18 & 0.95 & 0.07 \\
\hline Glutamate + glutamine & 6 & $473 \cdot 0$ & 439.9 & $476 \cdot 7$ & $17 \cdot 56$ & 0.51 & 0.62 & 0.30 \\
\hline Hydroxyproline & 6 & $25 \cdot 3$ & $20 \cdot 7$ & $23 \cdot 0$ & 1.88 & 0.53 & 0.34 & 0.56 \\
\hline Ornithine & 6 & $133 \cdot 7$ & $110 \cdot 8$ & $110 \cdot 7$ & 7.07 & 0.20 & 0.08 & 0.99 \\
\hline Proline & 6 & $99 \cdot 0$ & 89.2 & $101 \cdot 3$ & $3 \cdot 18$ & 0.26 & 0.56 & 0.13 \\
\hline Serine & 6 & $116 \cdot 4$ & $101 \cdot 8$ & $118 \cdot 1$ & 4.56 & 0.08 & 0.31 & 0.04 \\
\hline Taurine & 6 & 38.0 & 41.4 & $50 \cdot 2$ & $5 \cdot 25$ & 0.30 & 0.27 & 0.28 \\
\hline Tyrosine & 6 & $97 \cdot 1$ & 97.4 & $101 \cdot 8$ & 4.51 & 0.90 & 0.81 & 0.71 \\
\hline
\end{tabular}

C, control treatment; P1, 0.23 M-propionate treatment; P2, 0.41 M-propionate treatment; EAA, essential amino acids; NEAA, non-essential amino acids. ${ }^{*}$ For details of diets and procedures, see p. 940.

† Contrast 1: C v. (P1+P2), contrast 2: P1 v. P2.

Table 3. Splanchnic and hindlimb net fluxes of amino acids $(\mathrm{mmol} / \mathrm{h})$ in lambs fed frozen rye grass with intraruminal infusions of propionate

(Mean values and standard errors of the mean)

\begin{tabular}{|c|c|c|c|c|c|c|c|c|}
\hline & \multirow[b]{2}{*}{$n$} & \multicolumn{3}{|c|}{ Treatments } & \multirow[b]{2}{*}{ SEM } & \multirow[b]{2}{*}{ Treatment effect } & \multicolumn{2}{|c|}{ Orthogonal contrasts $†$} \\
\hline & & C & P1 & P2 & & & Propionate & Level \\
\hline \multicolumn{9}{|l|}{ TAA } \\
\hline PDV & 6 & 13.45 & $10 \cdot 65$ & $15 \cdot 87$ & 1.66 & 0.30 & 0.94 & 0.13 \\
\hline Liver & 6 & -12.83 & $-11 \cdot 17$ & -16.69 & 1.33 & 0.12 & 0.61 & 0.05 \\
\hline TSP & 6 & 0.62 & -0.53 & -0.82 & 1.40 & 0.86 & 0.60 & 0.92 \\
\hline $\mathrm{HL}$ & 4 & -0.78 & -0.95 & -0.51 & $0 \cdot 16$ & 0.45 & 0.93 & 0.23 \\
\hline \multicolumn{9}{|l|}{ EAA } \\
\hline PDV & 6 & $6 \cdot 38$ & 6.09 & $8 \cdot 17$ & 0.63 & 0.30 & 0.53 & 0.16 \\
\hline Liver & 6 & -2.72 & -3.60 & -4.87 & 0.48 & 0.07 & 0.06 & 0.15 \\
\hline TSP & 6 & 3.66 & 2.50 & $3 \cdot 31$ & 0.64 & 0.69 & 0.53 & 0.56 \\
\hline $\mathrm{HL}$ & 4 & -0.64 & -0.64 & -0.35 & $0 \cdot 10$ & 0.21 & 0.39 & $0 \cdot 13$ \\
\hline \multicolumn{9}{|l|}{ NEAA } \\
\hline PDV & 6 & 7.08 & 4.56 & $7 \cdot 70$ & 1.14 & 0.37 & 0.63 & 0.19 \\
\hline Liver & 6 & $-10 \cdot 11$ & -7.58 & -11.82 & 0.94 & 0.12 & 0.80 & 0.05 \\
\hline TSP & 6 & -3.04 & -3.02 & $-4 \cdot 13$ & 0.85 & 0.71 & 0.69 & 0.48 \\
\hline $\mathrm{HL}$ & 4 & -0.14 & -0.31 & $-0 \cdot 16$ & 0.08 & 0.85 & 0.74 & 0.68 \\
\hline \multicolumn{9}{|l|}{ BCAA } \\
\hline PDV & 6 & 2.52 & 2.41 & 4.05 & 0.34 & 0.11 & 0.31 & 0.06 \\
\hline Liver & 6 & 0.08 & -0.89 & -1.49 & 0.27 & 0.05 & 0.03 & 0.30 \\
\hline TSP & 6 & $2 \cdot 60$ & 1.52 & 2.56 & 0.37 & 0.40 & 0.47 & 0.26 \\
\hline $\mathrm{HL}$ & 4 & -0.43 & -0.41 & -0.22 & 0.07 & 0.17 & 0.27 & $0 \cdot 12$ \\
\hline
\end{tabular}

C, control treatment; P1, 0.23 M-propionate infusion; P2, 0.41 M-propionate infusion; TAA, total amino acids; PDV, portal-drained viscera; TSP, total splanchnic tissues; HL, hindlimb; EAA, essential amino acids; NEAA, non-essential amino acids; BCAA, branched-chain amino acids.

* For details of diets and procedures, see p. 940.

† Contrast 1: C v. (P1+P2), contrast 2: P1 v. P2. 
Table 4. Splanchnic and hindlimb net fluxes of essential amino acids $(\mathrm{mmol} / \mathrm{h})$ in lambs fed frozen rye grass with intraruminal infusion of propionate*

(Mean values and standard errors of the mean)

\begin{tabular}{|c|c|c|c|c|c|c|c|c|}
\hline & \multirow[b]{2}{*}{$n$} & \multicolumn{3}{|c|}{ Treatments } & \multirow[b]{2}{*}{ SEM } & \multirow[b]{2}{*}{ Treatment effect } & \multicolumn{2}{|c|}{ Orthogonal contrasts } \\
\hline & & C & P1 & P2 & & & Propionate & Level \\
\hline \multicolumn{9}{|l|}{ Histidine } \\
\hline PDV & 6 & 0.26 & 0.09 & 0.24 & 0.03 & 0.07 & 0.14 & 0.06 \\
\hline Liver & 6 & -0.11 & -0.14 & -0.14 & 0.03 & 0.94 & 0.73 & 0.95 \\
\hline TSP & 6 & 0.15 & -0.05 & 0.10 & 0.04 & 0.24 & 0.23 & 0.23 \\
\hline $\mathrm{HL}$ & 4 & -0.01 & -0.02 & -0.00 & 0.00 & 0.65 & 0.94 & 0.38 \\
\hline \multicolumn{9}{|c|}{ Isoleucine } \\
\hline PDV & 6 & 0.72 & 0.71 & 1.22 & 0.11 & $0 \cdot 13$ & 0.29 & 0.08 \\
\hline Liver & 6 & 0.00 & -0.26 & -0.39 & 0.07 & $0 \cdot 10$ & 0.05 & 0.45 \\
\hline TSP & 6 & 0.72 & 0.45 & 0.83 & 0.12 & 0.33 & 0.71 & 0.16 \\
\hline $\mathrm{HL}$ & 4 & $-0 \cdot 12$ & $-0 \cdot 13$ & -0.07 & 0.02 & 0.34 & 0.64 & 0.18 \\
\hline \multicolumn{9}{|l|}{ Leucine } \\
\hline PDV & 6 & 1.61 & 1.28 & 1.73 & 0.12 & 0.33 & 0.67 & 0.16 \\
\hline Liver & 6 & -0.03 & -0.43 & -0.61 & 0.09 & 0.02 & 0.01 & 0.29 \\
\hline TSP & 6 & 1.58 & 0.85 & $1 \cdot 12$ & 0.15 & 0.04 & 0.02 & 0.29 \\
\hline $\mathrm{HL}$ & 4 & $-0 \cdot 12$ & -0.14 & -0.09 & 0.02 & 0.59 & 1.00 & 0.33 \\
\hline \multicolumn{9}{|l|}{ Lysine } \\
\hline PDV & 6 & $1 \cdot 14$ & $1 \cdot 12$ & 1.43 & 0.12 & 0.44 & 0.55 & 0.27 \\
\hline Liver & 6 & -0.23 & -0.64 & -0.87 & 0.09 & 0.01 & 0.01 & 0.20 \\
\hline TSP & 6 & 0.91 & 0.48 & 0.57 & 0.13 & 0.14 & 0.06 & 0.67 \\
\hline $\mathrm{HL}$ & 4 & -0.07 & $-0 \cdot 11$ & -0.07 & 0.01 & $0 \cdot 19$ & $0 \cdot 26$ & 0.14 \\
\hline \multicolumn{9}{|c|}{ Methionine } \\
\hline PDV & 6 & 0.41 & 0.25 & 0.34 & 0.05 & 0.15 & 0.11 & 0.25 \\
\hline Liver & 6 & -0.26 & -0.24 & -0.34 & 0.03 & 0.20 & 0.52 & 0.10 \\
\hline TSP & 6 & $0 \cdot 15$ & 0.01 & 0.00 & 0.03 & 0.03 & 0.01 & 0.93 \\
\hline $\mathrm{HL}$ & 4 & -0.01 & -0.02 & -0.02 & 0.00 & 0.32 & $0 \cdot 17$ & 0.71 \\
\hline \multicolumn{9}{|c|}{ Phenylalanine } \\
\hline PDV & 6 & 1.26 & 1.02 & 1.03 & 0.09 & 0.39 & 0.18 & 0.93 \\
\hline Liver & 6 & $-1 \cdot 20$ & $-1 \cdot 12$ & $-1 \cdot 15$ & 0.08 & 0.91 & 0.70 & 0.88 \\
\hline TSP & 6 & 0.06 & -0.10 & -0.11 & 0.07 & 0.38 & 0.18 & 0.93 \\
\hline $\mathrm{HL}$ & 4 & -0.02 & -0.03 & -0.01 & 0.01 & 0.48 & $0 \cdot 70$ & 0.28 \\
\hline \multicolumn{9}{|c|}{ Threonine } \\
\hline PDV & 6 & 0.79 & 1.20 & 1.07 & 0.15 & 0.48 & 0.26 & 0.71 \\
\hline Liver & 6 & -0.99 & -0.56 & -0.88 & 0.15 & 0.24 & 0.23 & 0.22 \\
\hline TSP & 6 & -0.20 & 0.64 & 0.19 & 0.16 & 0.07 & 0.05 & 0.18 \\
\hline $\mathrm{HL}$ & 4 & $-0 \cdot 10$ & -0.07 & -0.03 & 0.02 & 0.30 & 0.15 & 0.74 \\
\hline \multicolumn{9}{|l|}{ Valine } \\
\hline PDV & 6 & 0.18 & 0.42 & 1.11 & 0.15 & 0.04 & 0.06 & 0.05 \\
\hline Liver & 6 & 0.11 & -0.20 & -0.50 & 0.14 & 0.24 & 0.15 & 0.39 \\
\hline TSP & 6 & 0.29 & 0.22 & 0.61 & 0.16 & 0.69 & 0.77 & 0.43 \\
\hline $\mathrm{HL}$ & 4 & -0.19 & -0.15 & -0.07 & 0.03 & 0.01 & 0.01 & 0.02 \\
\hline
\end{tabular}

C, control treatment; P1, 0.23 M-propionate infusion; P2, 0.41 M-propionate infusion; PDV, portal-drained viscera; TSP, total splanchnic tissues; $\mathrm{HL}$, hindlimb.

* For details of diets and procedures, see p. 940.

†Contrast 1: C v. (P1+P2), contrast 2: P1 v. P2.

$(P<0.13)$ increased with the propionate infusion, and especially for the P2 treatment (Table 4$)$. When considering NEAA, the propionate infusion led to significant increases in the NPA of tyrosine $(P<0 \cdot 04)$ and decreases in the NPA of aminobutyric acid $(P<0.09)$, cystathionine $(P<0.01)$, glycine $(P<0.07)$ and Glx $(P<0.04)$ (Table 5). Except for histidine and serine, whose NPA was decreased in the P1-treated animals (Tables 4 and 5) in comparison with the control and P2-treated animals, the NPA of the AA for the P1 animals was intermediate between that for the control and P2 animals. An important effect of the propionate infusion was an increase (approximately 2-fold) in the net transfer of arterial plasma urea-N into the lumen of the gut $(P<0.06)$ with no differences between propionate concentrations (data not shown).

Net fluxes of amino acids in the liver and the splanchnic tissues

Control animals. Across the liver, net uptake of AA occurred. For the control animals, $95 \%$ of the NPA of TAA-N was removed by the liver (Table 3 ). The net uptake was more limited for the EAA-N (43\% of NPA on average, and $3 \%$ for the BCAA-N) than for the NEAA-N (143\% of their NPA), implying a large uptake of the arterial NEAA-N also. Taken individually, the net 
Table 5. Splanchnic and hindlimb net fluxes of non-essential amino acids ( $\mathrm{mmol} / \mathrm{h}$ ) in lambs fed frozen rye grass with intraruminal infusions of propionate ${ }^{\star}$

(Mean values and standard errors of the mean)

\begin{tabular}{|c|c|c|c|c|c|c|c|c|}
\hline & \multirow[b]{2}{*}{$n$} & \multicolumn{3}{|c|}{ Treatments } & \multirow[b]{2}{*}{ SEM } & \multirow[b]{2}{*}{ Treatment effect } & \multicolumn{2}{|c|}{ Orthogonal contrasts $\dagger$} \\
\hline & & $\mathrm{C}$ & $\mathrm{P} 1$ & P2 & & & Propionate & Level \\
\hline \multicolumn{9}{|l|}{ Alanine } \\
\hline PDV & 6 & 1.70 & 2.57 & 3.05 & 0.35 & 0.13 & 0.06 & 0.45 \\
\hline Liver & 6 & -2.48 & -2.85 & -3.58 & 0.24 & 0.07 & 0.07 & 0.11 \\
\hline TSP & 6 & -0.78 & -0.28 & -0.53 & 0.18 & 0.48 & 0.30 & 0.54 \\
\hline $\mathrm{HL}$ & 4 & -0.045 & -0.05 & -0.00 & 0.02 & 0.77 & 0.84 & 0.52 \\
\hline \multicolumn{9}{|c|}{ Aminobutyric acid } \\
\hline PDV & 6 & 0.04 & -0.02 & 0.01 & 0.02 & 0.09 & 0.06 & 0.25 \\
\hline Liver & 6 & 0.02 & -0.02 & -0.01 & 0.03 & 0.57 & 0.34 & 0.68 \\
\hline TSP & 6 & 0.06 & -0.04 & 0.00 & 0.02 & 0.14 & 0.08 & 0.34 \\
\hline $\mathrm{HL}$ & 4 & 0.00 & 0.00 & 0.00 & 0.00 & 0.96 & $0 \cdot 81$ & 0.92 \\
\hline \multicolumn{9}{|l|}{ Arginine } \\
\hline PDV & 6 & 0.04 & -0.03 & 0.33 & $0 \cdot 11$ & 0.19 & 0.52 & 0.09 \\
\hline Liver & 6 & -0.85 & -0.76 & $-1 \cdot 12$ & 0.20 & 0.59 & 0.77 & 0.34 \\
\hline TSP & 6 & -0.80 & -0.79 & -0.79 & 0.14 & 1.00 & 0.95 & 1.00 \\
\hline $\mathrm{HL}$ & 4 & -0.05 & -0.07 & -0.04 & 0.01 & 0.80 & 0.89 & 0.54 \\
\hline \multicolumn{9}{|c|}{ Asparagine + aspartate } \\
\hline PDV & 6 & 0.75 & 0.41 & 1.33 & 0.20 & 0.11 & 0.74 & 0.04 \\
\hline Liver & 6 & $-1 \cdot 15$ & -0.64 & $-1 \cdot 22$ & 0.21 & 0.31 & 0.52 & 0.17 \\
\hline TSP & 6 & -0.40 & -0.23 & $0 \cdot 10$ & $0 \cdot 16$ & 0.03 & 0.03 & 0.06 \\
\hline $\mathrm{HL}$ & 4 & -0.05 & -0.11 & -0.02 & 0.02 & 0.04 & 0.29 & 0.02 \\
\hline \multicolumn{9}{|c|}{ Citrulline } \\
\hline PDV & 6 & 0.30 & 0.19 & 0.57 & 0.12 & 0.38 & 0.73 & 0.19 \\
\hline Liver & 6 & -0.13 & 0.21 & -0.34 & $0 \cdot 12$ & 0.18 & 0.79 & 0.07 \\
\hline TSP & 6 & 0.17 & 0.40 & 0.23 & 0.11 & 0.71 & 0.58 & 0.56 \\
\hline $\mathrm{HL}$ & 4 & -0.05 & -0.04 & -0.01 & 0.01 & $0 \cdot 71$ & 0.47 & $0 \cdot 76$ \\
\hline \multicolumn{9}{|c|}{ Cystathionine } \\
\hline PDV & 6 & 0.03 & -0.03 & -0.02 & 0.01 & 0.01 & 0.00 & 0.82 \\
\hline Liver & 6 & -0.02 & 0.00 & 0.02 & 0.01 & 0.06 & 0.04 & 0.16 \\
\hline TSP & 6 & 0.01 & -0.03 & -0.01 & 0.01 & 0.12 & 0.08 & 0.23 \\
\hline $\mathrm{HL}$ & 4 & 0.00 & 0.00 & 0.00 & 0.00 & 0.40 & 0.24 & 0.56 \\
\hline \multicolumn{9}{|l|}{ Glycine } \\
\hline PDV & 6 & $2 \cdot 85$ & 0.65 & 1.64 & 0.41 & 0.07 & 0.04 & 0.25 \\
\hline Liver & 6 & -3.02 & $-2 \cdot 34$ & $-3 \cdot 78$ & 2.95 & 0.24 & 0.95 & 0.10 \\
\hline TSP & 6 & -0.17 & -1.69 & $-2 \cdot 14$ & 0.39 & 0.08 & 0.03 & 0.58 \\
\hline $\mathrm{HL}$ & 4 & $0 \cdot 13$ & 0.05 & 0.08 & 0.04 & 0.93 & 0.81 & 0.80 \\
\hline \multicolumn{9}{|c|}{ Glutamine + glutamate } \\
\hline PDV & 6 & -1.08 & $-2 \cdot 11$ & -2.05 & 0.24 & 0.04 & 0.01 & 0.89 \\
\hline Liver & 6 & 0.52 & $2 \cdot 12$ & 1.43 & 0.35 & 0.16 & 0.09 & 0.37 \\
\hline TSP & 6 & -0.56 & 0.02 & -0.62 & 0.31 & 0.63 & 0.69 & 0.40 \\
\hline $\mathrm{HL}$ & 4 & -0.02 & 0.04 & -0.06 & 0.03 & 0.63 & 0.99 & 0.37 \\
\hline \multicolumn{9}{|c|}{ Hydroxyproline } \\
\hline PDV & 6 & -0.09 & -0.05 & 0.02 & 0.05 & 0.63 & 0.44 & 0.58 \\
\hline Liver & 6 & -0.18 & -0.22 & -0.08 & 0.05 & 0.08 & 0.59 & 0.03 \\
\hline TSP & 6 & -0.27 & -0.27 & -0.06 & 0.06 & 0.24 & 0.38 & 0.15 \\
\hline $\mathrm{HL}$ & 4 & 0.01 & -0.00 & 0.01 & 0.00 & 0.72 & 0.95 & 0.45 \\
\hline Ornithine & & & & & & & & \\
\hline PDV & 6 & 0.17 & 0.23 & 0.37 & 0.06 & 0.47 & 0.37 & 0.41 \\
\hline Liver & 6 & 0.39 & 0.60 & 0.24 & $0 \cdot 11$ & 0.27 & 0.88 & 0.12 \\
\hline TSP & 6 & 0.56 & 0.84 & 0.61 & 0.09 & 0.40 & 0.39 & 0.30 \\
\hline $\mathrm{HL}$ & 4 & -0.09 & -0.05 & -0.02 & 0.01 & 0.04 & 0.02 & 0.25 \\
\hline Proline & & & & & & & & \\
\hline PDV & 6 & 0.77 & 0.70 & 0.78 & 0.11 & 0.92 & 0.88 & 0.71 \\
\hline Liver & 6 & -0.55 & -0.73 & -0.61 & 0.08 & 0.64 & 0.49 & 0.52 \\
\hline TSP & 6 & 0.22 & -0.03 & 0.18 & 0.09 & 0.29 & 0.32 & 0.22 \\
\hline $\mathrm{HL}$ & 4 & -0.00 & -0.02 & -0.02 & 0.01 & $0 \cdot 17$ & 0.08 & 0.61 \\
\hline Serine & & & & & & & & \\
\hline PDV & 6 & 1.65 & 1.22 & 1.88 & 0.16 & 0.10 & 0.66 & 0.04 \\
\hline Liver & 6 & -1.86 & -1.68 & -1.81 & 0.10 & 0.33 & 0.28 & 0.30 \\
\hline TSP & 6 & -0.21 & -0.46 & 0.07 & 0.14 & 0.25 & 0.96 & 0.10 \\
\hline $\mathrm{HL}$ & 4 & -0.11 & -0.12 & -0.09 & 0.02 & 0.81 & 0.83 & 0.57 \\
\hline
\end{tabular}


Table 5. Continued

\begin{tabular}{|c|c|c|c|c|c|c|c|c|}
\hline & \multirow[b]{2}{*}{$n$} & \multicolumn{3}{|c|}{ Treatments } & \multirow[b]{2}{*}{ SEM } & \multirow[b]{2}{*}{ Treatment effect } & \multicolumn{2}{|c|}{ Orthogonal contrasts $\dagger$} \\
\hline & & C & $\mathrm{P} 1$ & P2 & & & Propionate & Level \\
\hline \multicolumn{9}{|l|}{ Taurine } \\
\hline PDV & 6 & 0.43 & 0.33 & 0.26 & 0.04 & 0.40 & 0.22 & 0.60 \\
\hline Liver & 6 & -0.27 & -0.44 & -0.51 & 0.09 & 0.47 & 0.26 & 0.71 \\
\hline TSP & 6 & 0.16 & -0.11 & -0.25 & 0.10 & 0.11 & 0.05 & 0.45 \\
\hline $\mathrm{HL}$ & 4 & 0.03 & -0.02 & -0.12 & 0.14 & 0.09 & 0.04 & 0.47 \\
\hline \multicolumn{9}{|c|}{ Tyrosine } \\
\hline PDV & 6 & 0.39 & 0.67 & 0.74 & 0.08 & 0.04 & 0.02 & 0.57 \\
\hline Liver & 6 & -0.72 & -0.72 & -1.14 & 0.09 & 0.12 & 0.27 & 0.08 \\
\hline TSP & 6 & -0.33 & -0.05 & -0.40 & 0.09 & 0.04 & 0.32 & 0.02 \\
\hline $\mathrm{HL}$ & 4 & -0.01 & -0.02 & 0.00 & 0.01 & 0.31 & 0.82 & 0.15 \\
\hline
\end{tabular}

C, control treatment; P1, 0.23 M-propionate infusion; P2, 0.41 M-propionate infusion; PDV, portal-drained viscera; TSP, total splanchnic tissues; $\mathrm{HL}$, hindlimb.

${ }^{*}$ For details of diets and procedures, see p. 940.

† Contrast 1: C v. (P1+P2), contrast 2: P1 v. P2.

hepatic flux of AA followed the same pattern as that of TAA, except for Glx, which showed a net hepatic release $(0.52(\operatorname{SEM} 0.35) \mathrm{mmol} / \mathrm{h})$. In terms of EAA, fractional extraction (Table 6) was greatest for phenylalanine $(16.3 \%)$ and methionine $(10.4 \%)$, moderate for threonine $(6.1 \%)$ and minor for the other AA. As for the NEAA, fractional extractions were generally higher, especially for aspartate + asparagine $(15.2 \%)$, serine $(17.6 \%)$ and alanine $(11.9 \%)$. The majority of the $\mathrm{NH}_{3}-\mathrm{N}$ appearing in the portal vein $(96 \%)$ was taken up by the liver.

Propionate effects. The effects of propionate on the net hepatic fluxes of AA were not always clear-cut because of variations in response to the different levels of propionate. Briefly, the net hepatic uptake of EAA, in particular the BCAA (isoleucine $(P<0 \cdot 10)$ and leucine $(P<0.02)$ ), lysine $(P<0.01)$ and methionine $(P<0 \cdot 10)$ (for $\mathrm{P} 2$ only), increased in a dose-dependent manner (Table 4). This effect was associated with increases in the fractional extraction of these AA (Table 6). The net uptake of the gluconeogenic alanine tended to increase by $15 \%(\mathrm{P} 1)$ and $44 \%(\mathrm{P} 2)(P<0.07)$ as a consequence of the higher
NPA, since the hepatic fractional extraction was not modified. The net hepatic release of Glx tended to increase $(P<0.09)$ with the propionate infusion, but in this case the effect was associated with a change in the hepatic fractional extraction.

The net splanchnic release of leucine $(P<0.02)$ and methionine $(P<0.01)$ was reduced by the propionate treatment, whereas that of threonine tended to increase $(P<0.05)$ (Table 4). Of the NEAA, propionate supplementation did not clearly influence their splanchnic release (Table 5). The net hepatic uptake of $\mathrm{NH}_{3}-\mathrm{N}$ was not significantly modified by the propionate infusion, but net hepatic urea-N release tended to be increased with the $\mathrm{P} 1$ treatment $(P<0 \cdot 08)$ (data not shown).

\section{Net fluxes of amino acids in the hindlimb}

Control animals. In the hindlimb, AA were extracted $(-0.78 \mathrm{mmol} / \mathrm{h}$ for TAA $)$, mostly as EAA $(-0.64 \mathrm{mmol} /$ h) (Table 3). Taken individually, all AA were removed by the hindlimb (this is the case for isoleucine, leucine,

Table 6. Liver extraction rates (\%) of amino acids in lambs fed frozen rye grass with intraruminal infusions of propionate* (Mean values and standard errors of the mean)

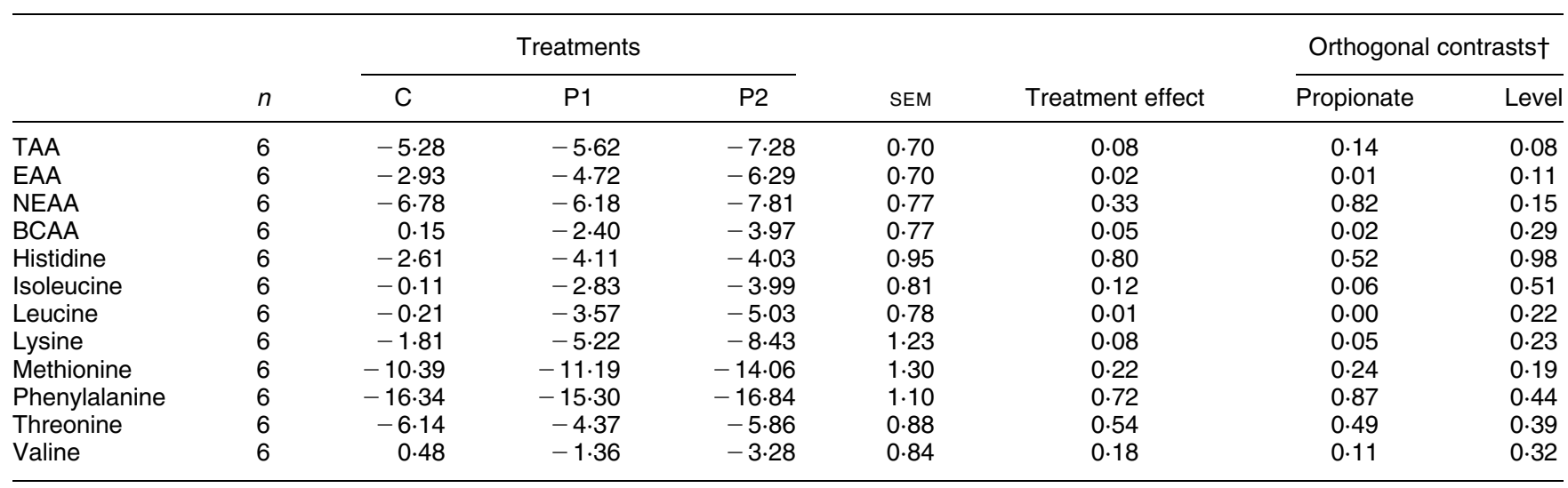

C, control treatment; P1, 0.23 M-propionate infusion; P2, 0.41 M-propionate infusion; TAA, total amino acids; EAA, essential amino acids; NEAA, non-essential amino acids; BCAA, branched-chain amino acids.

${ }^{*}$ For details of diets and procedures, see p. 940.

† Contrast 1: C v. (P1+P2), contrast 2: P1 v. P2. 
lysine, threonine, valine, arginine, citrulline, ornithine and serine) or not removed (AA whose net balance was not significantly different from zero: histidine, methionine, phenylalanine, alanine, aminobutyric acid, aspartate + asparagine, cystathionine, Glx, hydroxyproline, proline, taurine and tyrosine) (Tables 4 and 5). Glycine is the only AA that showed a net release $(0.13 \mathrm{mmol} / \mathrm{h})$.

Propionate effects. No major effect of the propionate treatment was observed for the net AA fluxes (TAA, EAA and NEAA) across the hindlimb (Table 3). Indeed, the decreases in net splanchnic release mentioned earlier were partially compensated by increased fractional extraction in the hindlimb (for example, methionine $(P<0.07)$ and lysine $(P<0 \cdot 05))$ (Table 4$)$. Valine uptake decreased $(P<0.01)$ after the propionate infusion (Table 4$)$. The net hindlimb fluxes were not altered by the propionate infusion for the other AA, except for taurine and ornithine (Table 5).

\section{Discussion}

The aim of the present study was to investigate how an alteration of the VFA profile in the rumen (with an increase in the propionate:acetate ratio) could alter the utilisation of the $\mathrm{N}$ nutrients (AA, $\mathrm{NH}_{3}$ and urea) by tissues and organs such as the digestive tract, the liver and muscle. The effect of an increased energy supply on the protein metabolic processes (direct effect) or via some sparing processes of the AA by the energetic compounds (indirect effect) was also investigated. Concerning the sparing effect of the AA by the energetic compounds, a significant proportion of the AA absorbed by the splanchnic tissues is used for purposes other than protein accretion. Some NEAA (such as alanine, glutamate and glutamine; Gate et al. 1999) and EAA (such as leucine; Lapierre et al. 1997) are oxidised in the PDV to sustain its energy requirements. The AA are also involved in urea synthesis (for $\mathrm{NH}_{3}$ detoxification) and gluconeogenesis in the liver. Consequently, the fate of the AA in the splanchnic tissues (which, as previously stated, are complex and involve various metabolic routes) will influence the AA supply to the peripheral tissues such as muscle and will have an effect on growth.

Part of the research programme, of which the present study is also a part, was focused on the effects of an intraruminal propionate infusion on energy metabolism as well as the utilisation and metabolism of the energy-yielding nutrients (VFA, glucose, lactate) across the ruminant tissues. These results are already published (Majdoub et al. 2003).

\section{Absorption and utilisation of the nitrogenous compounds by the portal-drained viscera}

The NPA of $\mathrm{N}$ (as $\mathrm{NH}_{3}-\mathrm{N}$ and TAA-N) averaged 30-40\% of the total $\mathrm{N}$ intake throughout the treatments (control, P1 and $\mathrm{P} 2$ ). This ratio takes into account the utilisation of $\mathrm{N}$ by the digestive tract and underestimates the apparent $\mathrm{N}$ digestibility. Despite this known underestimation, the ratio that was obtained was still low compared with the digestibility values normally found with a fresh rye grass, namely, 70-75\% (Institut National de Recherches Agronomiques, 1978). The NPA of $\mathrm{NH}_{3}-\mathrm{N}$ was very low in the present experiment $(13-17 \% \mathrm{~N}$ intake) as in the preliminary trial (also using frozen grass) (Majdoub et al. 1999). The NPA of $\mathrm{NH}_{3}-\mathrm{N}$ was low compared with the values generally obtained in ruminant animals (30-80\%; Huntington et al. 1996), especially when fed fresh grass or grass silage (34-49\%; Journet et al. 1995; DeVisser et al. 1997). The measurement of $\mathrm{NH}_{3}-\mathrm{N}$ in the plasma instead of in the blood in the present study could have induced an underestimation of its NPA (Nozière et al. 1998). On the contrary, NPA of TAA-N is consistent with that obtained in the literature (about $20 \% \mathrm{~N}$ intake) but lower than previously measured in younger lambs (57\%; Majdoub et al. 1999). The net positive release of TAA by the PDV $(13.45 \mathrm{mmol} / \mathrm{h})$ of the control animals is consistent with what has previously been observed in sheep $(46 \mathrm{~kg})$ fed $1.25 \times$ maintenance with a pelleted cereal grain-straw diet $(17.46 \mathrm{mmol} / \mathrm{h}$; Balcells et al. 1995). This release of TAA from the gut tissues is normally expected in animals fed above maintenance (Huntington et al. 1988; Van der Walt, 1993; Lapierre et al. 2000). A comparison of the AA appearing in the portal vein in the control lambs given frozen rye grass with the composition of rumen microbial protein (Storm \& Ørskov, 1983) was made and the values were normalised relative to leucine. Except for valine (and to a lesser extent threonine), the AA profile of the EAA was in agreement with that reported for rumen microbial protein (Van der Walt, 1993; Lobley et al. 1996a). The deficit in the apparent uptake of valine and threonine in the present study can be explained by the relatively important proportion of these two AA in the proteins secreted by the gut tissues, such as mucins (Mukkur et al. 1985; Lien et al. 1997), which are poorly reabsorbed by the hindgut and consequently lost in the faeces. In summary, the freezing process of the rye grass alters the $\mathrm{N}$ solubility of the feed but whether or not the freezing increases or decreases the $\mathrm{N}$ digestibility remains a matter of debate (MacRae et al. 1975; Hristov, 1998). These results indicate that the absolute values of $\mathrm{N}$ absorption and utilisation by the PDV obtained in the present study with frozen rye grass cannot be extrapolated to fresh grass. The AA pattern absorbed by the digestive tract is very close to that of rumen microbial protein, showing that a small quantity of AA arising directly from the diet actually reach the intestine. These AA are consequently extensively utilised and metabolised by the rumen microflora before reaching the intestine.

The EAA release by the PDV represented approximately $50 \%$ of the release of the TAA and was not strongly altered by the propionate treatments $(47,57$ and $51 \%$ for the control, P1 and P2 animals respectively). This proportion has also been shown to be unresponsive to intake level (Lapierre et al. 2000). However, the propionate infusion induced an increase in the PDV release of BCAA $(+61 \%)$ (valine especially) and tyrosine, which is consistent with the results from Nozière et al. (2000) in underfed ewes infused with VFA. Seal et al. (1993) also showed an increase in portal BCAA concentrations in lambs fed hay for a growth of $100 \mathrm{~g} / \mathrm{d}$ and infused with propionate at a concentration equivalent to the $\mathrm{P} 1$ treatment in the present experiment. This suggests a decrease in the utilisation and catabolism of these AA by the PDV and a greater availability for other tissues and organs (Seal et al. 1993). Hence, 
an indirect effect of the propionate infusion on the BCAA catabolism in the PDV can be hypothesised. The mechanisms responsible for the sparing effect of propionate on the BCAA can be explained by either an alteration of the rumen microbiota or a decreased utilisation of these AA by the gut cells.

Concerning the NEAA, glutamate and glutamine are removed by the PDV for energetic purposes (Gate et al. 1999; Reeds et al. 2000; Lobley et al. 2001; Reeds \& Burrin, 2001; for a review, see Van der Walt, 1993), and in rodents, are partly transaminated to alanine $33 \%$ of the glutamine uptake; Windmueller, 1982). Glutamine also plays an important role in EAA protection from oxidation (and could explain the increased NPA of BCAA, as shown earlier) (Lobley et al. 2001). The propionate infusion induced an increase in the Glx net uptake by the PDV, which was consistent with the numerical increase in the net PDV release of alanine $(+79 \%$ between the control and P2 treatments; $P<0.13$ ). These results differ from those of Seal \& Parker (1996) and Nozière et al. (2000), where the propionate infusion had no effect on the net PDV release or uptake of alanine, glutamate and glutamine, despite similar propionate infusion rates. The infusion rates were $1 \mathrm{~mol} / \mathrm{d}$ in the reported studies $v .0 .55$ and $0.98 \mathrm{~mol} / \mathrm{d}$ for the P1 and P2 treatments respectively in the present study. The reasons for this discrepancy are unclear. First, the rumen microbes seem to use important amounts of propionate (Kristensen et al. 2000). In the present study the propionate infusion may have altered the profile of the nutrients used by the rumen microbes and have spared some AA such as glutamate and glutamine. This statement is reinforced by the fact that an increased urea net transfer from the arterial blood to the gut has been observed after the propionate infusion. Second, the butyrate concentration in the rumen fluid tended to be lower with the propionate infusion in the present study, as previously noted by Moloney (1998), suggesting a small drop in the protozoal population in the rumen (Journet et al. 1995). A modification in the profile of the rumen microbes can lead to an altered profile in the AA and energy nutrients supplied to the intestine and possibly in the AA profile appearing in the portal vein. Concerning the utilisation of the propionate as a substrate by the gastrointestinal cells themselves, propionate is known to increase the proliferation of epithelial cells in vivo (Sakata \& Tamate, 1978) with a more complex action in vitro (Baldwin, 1999). Glutamine is one of the possible precursors for mucosal nucleic acid synthesis (Newsholme \& Carrie, 1994; Gate et al. 1999; Reeds et al. 1999). Hence, it is possible that a stimulation of cell proliferation by propionate could be partly responsible for the increased net PDV Glx uptake (driven by an increased glutamine uptake).

Another NEAA whose PDV release tended to be modified (decreased; $P<0.07$ ) by the treatment was glycine. Rémond $(2000 a, b)$ showed that the rumen can be a net user of glycine. Consequently, with the assumed modifications in rumen wall metabolism due to the propionate infusion, the removal of glycine by the rumen could have become more important in glycine utilisation in comparison with the rest of the PDV. The involvement of glycine in nucleic acid production may also be responsible for the alterations in its net PDV release.

\section{Absorption and utilisation of the nitrogenous compounds by the liver}

The quantity of TAA-N utilised by the liver represented about $10-20 \%$ of the ingested $\mathrm{N}$ in the control animals. This is in accordance to what was observed by Reynolds et al. (1991), where 40-70\% of the NPA of TAA-N was removed by the liver. The fate of the AA in the liver can be 2-fold: (1) oxidation for energetic purposes and ureagenesis in parallel or (2) synthesis of endogenous or exported proteins in the liver (Lobley, 1994). The quantity of TAA-N utilised by the liver was increased after the propionate treatments ( 20 and $31 \%$ of $\mathrm{N}$ intake for the control and $\mathrm{P} 2$ treatments respectively). For the $\mathrm{P} 1$ and $\mathrm{P} 2$ animals, the TAA-N uptake by the liver was even greater than the TAA-N appearing in the portal vein, showing a contribution of the arterial supply of AA to the liver in this situation. To explain this result, several hypotheses can be made. These are: (1) the peripheral tissues (such as muscle) are in a catabolic state to supply AA to the liver; (2) the liver synthesises more exported proteins, which have not been measured in the present study; or (3) some amino- $\mathrm{N}$ has not been taken into account in the measurements in the present study (such as peptides). No effect of the propionate treatment on the hindlimb net TAA balance could be demonstrated; however, the net BCAA uptake by the hindlimb tended to decrease $(P<0.07)$ after the propionate infusion. Hence, an increased supply of AA from the peripheral tissues to the liver (via arterial blood) cannot be excluded during the propionate infusion. An increase in the synthesis of liver proteins can also be hypothesised, probably due to the increased supply of energy to the liver (Lobley et al. 1999). The utilisation of the TAA-N by the liver is increased after the propionate treatments in parallel with the increased insulin secretion and uptake by the liver measured in those same animals (Majdoub et al. 2003). An increased insulin secretion was previously reported after propionate treatment by Harmon (1992), Seal \& Parker (1994) and Casse et al. (1994). While no obvious effect of insulin on protein synthesis in the liver has been demonstrated (Ahlman et al. 2001), insulin does play a role in the synthesis of exported proteins. Insulin induces an increased synthesis of albumin (one of the major exported proteins) (De Feo et al. 1991; Ahlman et al. 2001) and a decreased synthesis of fibrinogen (De Feo et al. 1991; Ahlman et al. 2001). An increased synthesis of exported proteins induced by insulin may be one of the explanations for the increased AA uptake by the liver. Last, the potential role of the peptides is much more difficult to assess in the present study since there was no direct measurement of these moieties. According to Backwell et al. (1997) and Webb (1999), it seems that intact peptides, whether appearing in the portal vein (through the omasal epithelium) and utilised by the different tissues and organs, are not the major form for $\mathrm{N}$ transfer in the body. However, recent results from Rémond $(2000 a, b)$ showed that $38 \%$ of the total amino-N 
portal flux can be attributed to the peptides. Consequently, even if minor, the importance of these peptides may vary with the level of intake and the nature of the diet given to the animals.

EAA utilisation by the liver represented 21, 32 and $29 \%$ of the TAA taken up by the liver for the control, P1- and P2-treated animals respectively. Again, these values are consistent with the values obtained by Koeln et al. (1993; 34\%), Reynolds et al. (1994; 32\%), Bruckental et al. $(1997 ; 21 \%)$ and Lapierre et al. $(2000 ; 15 \%)$. In contrast to what was observed in the PDV, the propionate treatment (and especially P2) induced an increase in TAA utilisation by the liver $(+30 \%$ between the control and $\mathrm{P} 2$ treatment). This was mainly the case for EAA $(+79 \%)$ and to a lesser extent for NEAA $(+17 \%)$. The response of EAA gives another reason to suggest an increase in protein synthesis in the liver (Lobley et al. 1999). As previously discussed, the role of insulin in the protein synthesis of exported proteins (such as albumin) could play an important role in the utilisation of the EAA in the liver. The utilisation of NEAA was not strongly altered, except for alanine. Indeed, alanine uptake tended to increase by $43 \%(P<0.07)$. In vitro, propionate induced a decrease in alanine oxidation, which could be explained by a substitution of alanine by propionate for net glucose production (Mutsvangwa et al. 1997). However, in the present experiment, net hepatic glucose production was not altered (Majdoub et al. 2003), suggesting changes in the relative contribution of each glucose precursor. In the present case, the increased alanine uptake could compensate for a decreased hepatic utilisation of glutamate, which is commonly used in the liver as a gluconeogenic precursor (Van der Walt, 1993). For some specific AA (BCAA, alanine), the increase in the liver uptake occurred in parallel to the increase in PDV release. The liver responds by increasing its protein synthesis rate and inhibiting its protein degradation (Meijer et al. 1999) or by modifying the contribution of the different AA to gluconeogenesis. More specifically, the synthesis of exported proteins (such as serum albumin) is more reactive to a variation in the AA supply than the synthesis of endogenous hepatic proteins in single-stomached animals and in ruminants (Chiku et al. 1993; Barle et al. 1997; Connell et al. 1997). From the results obtained in the present study, the propionate infusion seems to have more influence on liver protein metabolism (probably via insulin) than on a sparing effect of propionate on AA utilisation by the liver (especially since no increased gluconeogenesis could be demonstrated when propionate was infused; Majdoub et al. 2003).

The propionate treatment did not have any strong effect on urea production by the liver. These results contrast with those obtained with hepatocytes in vitro in sheep (Demigné et al. 1991) or in rodents (Stewart \& Walser, 1980), where propionate induced an increased ureagenesis at a similar $\mathrm{NH}_{3}$ supply. However, these in vitro effects were not confirmed in vivo by Kim et al. (1999), where abomasal propionate infusion in adult sheep did not inhibit ureagenesis. However, the propionate concentration in the hepatic blood of our sheep ranged between 0.13 and $0.19 \mathrm{mM}$ for the control and P2 animals respectively
$(P<0.01)$ (Majdoub et al. 2003). This is much lower than the propionate concentrations used in the in vitro studies; $5 \mathrm{~mm}$ (Garwacki et al. 1990), and from 0.31 to $1.25 \mathrm{~mm}$ (Mutsvangwa et al. 1997).

Absorption and utilisation of the nitrogenous compounds by the splanchnic tissues (portal-drained viscera + liver)

Generally, the utilisation of specific AA by the liver (Biolo et al. 1992; Lobley et al. 1996b; Seal \& Parker, 1996) influences the AA profile offered to peripheral tissues, especially muscle. In the present experiment, the tendency for a greater release of AA (and especially the BCAA) by the PDV observed after the propionate treatment was entirely erased by the increase of the hepatic uptake of these AA. This resulted in no changes to the splanchnic AA balance by propionate.

With the propionate treatments, the net urea transfer from the artery to the gut increased. This is consistent with the data summarised by Rémond et al. (1996), where supplementation of the feed by rapidly fermentable energy sources doubled the daily flux of urea across the rumen wall (Norton et al. 1982; Theurer et al. 2002). Similar results were found in sheep (Kim et al. 1999) where propionate infusion in the abomasum induced a $40 \%$ increase in urea recycling. The mechanism by which propionate increases urea recycling may be an alteration of the rumen microbial metabolism (when fermentable carbohydrates are supplemented in the diet). The mechanism may be from an alteration of the urea entry across the rumen wall (when propionate itself is supplied in the rumen) or from an alteration in the utilisation of the propionate by the gut or the liver (when propionate is supplied in the abomasum) (Rémond et al. 1996; Kim et al. 1999; Kristensen et al. 2000). Another factor that can influence urea transfers between the blood and rumen is the $\mathrm{NH}_{3}-\mathrm{N}$ concentration present in the rumen (Ferrel et al. 2001), but in the present study it could not account for the increase in the urea transfer observed. Indeed, the $\mathrm{NH}_{3}-\mathrm{N}$ concentration in the rumen was not altered after the propionate treatment (Majdoub et al. 2003).

Surprisingly, and contrary to what was previously demonstrated by Seal \& Parker (1996), the increased urea net transfer from artery to the rumen after the propionate infusion did not increase the NPA of AA; in fact the NPA was decreased. However, because of the heterogeneity in the DM of the frozen grass given to the animals, the $\mathrm{N}$ intake in the control $(31.6 \mathrm{~g} / \mathrm{d})$ was higher $(P<0.05)$ than the $\mathrm{N}$ intake in the $\mathrm{P} 1(27.7 \mathrm{~g} / \mathrm{d})$ and $\mathrm{P} 2$ $(26.2 \mathrm{~g} / \mathrm{d})$ periods. This result was not due to a decrease in the total intake of frozen fresh grass after the propionate treatment but was totally explained by a higher DM of the grass given during the control period. When ingested $\mathrm{N}$ was used as a cofactor in the statistical analysis, this explained the decreased AA PDV net release $(P<0 \cdot 05)$. $\mathrm{NH}_{3}-\mathrm{N}$ and urea- $\mathrm{N}$ were measured in plasma, based on the Berthelot reaction (Berthelot, 1859) in the present experiment and not in blood. Thus an underestimation of the urea and $\mathrm{NH}_{3}$ fluxes occurred (Nozière et al. 1998). Consequently, it is difficult to interpret further the data concerning the urea- $\mathrm{N}$ and $\mathrm{NH}_{3}-\mathrm{N}$. 


\section{Nitrogen net uptake and release by the peripheral tissues (hindlimb)}

The effects of propionate (or glucose) treatments on $\mathrm{N}$ metabolism (global $\mathrm{N}$ retention, protein synthesis in muscle and milk production) in ruminants have been studied extensively (Eskeland et al. 1973, 1974; AbdulRazzaq \& Bickerstaffe, 1989; Seal et al. 1993; Huhtanen et al. 1998; Moloney, 1998; Kim et al. 1999; Ørskov et al. 1999; Obitsu et al. 2000). Whatever the mechanisms underlying this process (the role of insulin is indisputable), propionate or glucose infusions induce an increase in $\mathrm{N}$ retention in the muscle (Abdul-Razzaq \& Bickerstaffe, 1989; Moloney, 1998) or an increase in milk production and protein content in the milk (Huhtanen et al. 1998). However, in the present study, the propionate infusion had no effect on the net hindlimb uptake of TAA, EAA and NEAA. This is consistent with results at the splanchnic level, where no effect of the propionate treatment on the splanchnic release of AA was observed. Only the BCAA showed a tendency for a decrease in hindlimb uptake ( $-49 \%$ between the control and P2 animals). These results differed from what occurred for the energy nutrients. Despite an unchanged release of energy by the splanchnic tissues, the energy uptake by the hindlimb (as glucose, acetate and L-lactate) increased from 8 to 11 and $12 \mathrm{~kJ} / \mathrm{h}$ (Majdoub et al. 2003). An increased sensitivity and responsiveness of the hindlimb tissues to insulin has been hypothesised to explain the increased energy uptake. However, in terms of AA metabolism, the role of insulin is strongly correlated to the AA supply to the tissues (Grizard et al. 1995). In the present study, neither the AA nor the insulin supply to the hindlimb was altered by the propionate infusion. This could explain the lack of effect of the propionate treatment on the hindlimb net AA balance.

When looking at the AA concentrations in the artery, a decrease in EAA and BCAA concentrations $(-16$ and $-21 \%$ respectively between the control and P2 animals) occurred with the propionate treatment. Similar effects have been observed after glucose or propionate infusion in most studies (for example, Cappelli et al. 1997; Nozière et al. 2000; Obitsu et al. 2000), in spite of a few conflicting data where no change (Balcells et al. 1995) or an increase (Seal et al. 1993; Seal \& Parker, 1996) in EAA concentrations was observed. A decrease in AA concentrations in systemic blood would tend to indicate an increase in the utilisation of these AA by peripheral tissues (such as muscle), which was not confirmed by the net hindlimb fluxes measured.

In conclusion, although $\mathrm{N}$ utilisation by the digestive tract and the liver was altered by the propionate infusion (especially AA utilisation and release by the gut and the liver), no strong effect on the peripheral tissues could be demonstrated. In the present experiment, the regulation of metabolism in the splanchnic tissues (involving insulin and the nutrients themselves) had an effect on the relative potential contribution of each gluconeogenic precursor to the glucose synthesis in the liver without any strong effect on the energy and $\mathrm{N}$ supply to the peripheral tissues. However, even if no effect of propionate on muscle protein metabolism could be demonstrated, the propionate infusion induced an increased protein metabolism in the liver (via insulin). The close interaction between the liver and the muscle for AA utilisation plays a crucial role in the overall AA metabolism in the whole body.

\section{Acknowledgements}

The authors would like to express their deep appreciation to J. Lefaivre and D. Durand for their surgical expertise and to C. Leoty and R. Souchet for their excellent care of the animals. The technical expertise of A. IssertyThomas and C. Cossoul is gratefully recognised; Y. Colleaux is fully acknowledged for AA analysis.

\section{References}

Abdul-Razzaq HA \& Bickerstaffe R (1989) The influence of rumen volatile fatty acids on protein metabolism in growing lambs. Br J Nutr 62, 297-310.

Ahlman B, Charlton M, Fu A, Berg C, O'Brien P \& Nair KS (2001) Insulin's effect on synthesis rates of liver proteins. Diabetes 50, 947-954.

Backwell FRC, Hipolito-Reis M, Wilson D, Bruce LA, Buchan V \& MacRae JC (1997) Quantification of circulating peptides and assessment of peptide uptake across the gastrointestinal tract of sheep. J Anim Sci 75, 3315-3322.

Balcells J, Seal CJ \& Parker DS (1995) Effect of intravenous glucose infusion on metabolism of portal-drained viscera in sheep fed a cereal/straw-based diet. J Anim Sci 73, 2146-2155.

Baldwin RL (1999) The proliferative actions of insulin, insulinlike growth factor-I, epidermal growth factor, butyrate and propionate on ruminal epithelial cells in vitro. Small Rumin Res 32, 261-268.

Barle H, Nyberg B, Essen P, et al. (1997) The synthesis rates of total liver protein and plasma albumin determined simultaneously in vivo in humans. Hepatology 25, 154-158.

Barnes RJ, Comline RS \& Dobson A (1986) The control of splanchnic blood flow. In Control of Digestion and Metabolism in Ruminants, pp. 41-59 [LP Milligan, WL Grovum and A Dobson, editors]. Englewood Cliffs, NJ: Prentice Hall.

Bergmeyer HU (1985) Ammonia determination. In Methods of Enzymatic Analysis, vol. 8, p. 454 [HU Bergmeyer, J Bergmeyer and M Grassl, editors]. London: Academic Press.

Berthelot M (1859) Violet d'aniline (Aniline violet). Répertoire de Chimie Appliquée 1, 284.

Biolo G, Tessari P, Inchiostro S, et al. (1992) Leucine and phenylalanine kinetics during mixed meal ingestion: a multiple tracer approach. Am J Physiol 262, E455-E463.

Bruckental I, Huntington GB, Baer CK \& Erdman RA (1997) The effect of abomasal infusion of casein and recombinant somatotropin hormone injection on nitrogen balance and amino acid fluxes in portal-drained viscera and net hepatic and total splanchnic blood in Holstein steers. J Anim Sci 75, 1119-1129.

Cappelli FP, Seal CJ \& Parker DS (1997) Glucose and $\left[{ }^{13} \mathrm{C}\right]$ leucine metabolism by the portal-drained viscera of sheep fed on dried grass with acute intravenous and intraduodenal infusions of glucose. Br J Nutr 78, 931-946.

Casse EA, Rulquin H \& Huntington GB (1994) Effect of mesenteric vein infusion of propionate on splanchnic metabolism in primiparous holstein cows. J Dairy Sci 77, 3296-3303.

Chiku K, Mochida M, Yamamoto M \& Natori Y (1993) Amino acids suppress intracellular protein degradation in rat liver during parenteral nutrition. J Nutr 123, 1771-1776.

Connell A, Calder AG, Anderson SE \& Lobley GE (1997) Hepatic 
protein synthesis in the sheep: effect of intake as monitored by use of stable-isotope-labelled glycine, leucine and phenylalanine. Br J Nutr 77, 255-271.

De Feo P, Gaisano MG \& Haymond MW (1991) Differential effects of insulin deficiency on albumin and fibrinogen synthesis in humans. J Clin Invest 88, 833-840.

Demigné C, Yacoub C, Morand C \& Rémésy C (1991) Interactions between propionate and amino acid metabolism in isolated sheep hepatocytes. Br J Nutr 65, 301-317.

DeVisser H, Valk H, Klop A, Van der Meulen J, Bakker JGM \& Huntington GB (1997) Nutrient fluxes in splanchnic tissue of dairy cows: influence of grass quality. J Dairy Sci 80, 16661673.

Eskeland B, Pfander WH \& Preston RL (1973) Utilisation of volatile fatty acids and glucose for protein deposition in lambs. Br J Nutr 29, 347-355.

Eskeland B, Pfander WH \& Preston RL (1974) Intravenous energy infusion in lambs: effects on nitrogen retention, plasma free amino acids and plasma urea nitrogen. $\mathrm{Br} J$ Nutr 31, 201-211.

Ferrel CL, Freetly HC, Goetsch AL \& Kreikemeier KK (2001) The effect of dietary nitrogen and protein on feed intake, nutrient digestibility and nitrogen flux across the portal-drained viscera and liver of sheep consuming high-concentrate diets ad libitum. J Anim Sci 79, 1322-1328.

Garwacki S, Wiechetek M, Karlik W, Souffrant WB \& Krawielitzki K (1990) Effect of propionate on the utilization of nitrogen from ${ }^{15} \mathrm{NH}_{4} \mathrm{Cl}$ for urea synthesis in hepatocytes isolated from sheep liver. Int J Biochem 22, 1185-1188.

Gate JJ, Parker DS \& Lobley GE (1999) The metabolic fate of the amino-N group of glutamine in the tissues of the gastrointestinal tract in 24h-fasted sheep. Br J Nutr 81, 297-306.

Grizard J, Dardevet D, Papet I, et al. (1995) Nutrient regulation of skeletal muscle protein metabolism in animals. The involvement of hormones and substrates. Nutr Res Rev 8, 67-91.

Harmon DL (1992) Impact of nutrition on pancreatic exocrine and endocrine secretion in ruminants: a review. J Anim Sci 70, $1290-1301$.

Harmon DL \& Avery TB (1987) Effects of dietary monensin and sodium propionate on net nutrient flux in steers fed a high concentrate diet. J Anim Sci 65, 1610-1616.

Hovell FD \& Greenhalgh JFD (1978) The utilization of diets containing acetate, propionate or butyrate salts by growing lambs. Br J Nutr 40, 171-183.

Hristov AN (1998) Nitrogen fractions and in sacco dry matter and crude protein degradability of fresh and frozen alfalfa. Anim Feed Sci Technol 71, 351-355.

Huhtanen PJ, Blauwiekel R \& Saastamoinen I (1998) Effects of intraruminal infusions of propionate and butyrate with two different protein supplements on milk production and blood metabolites in dairy cows receiving grass silage-based diet. J Sci Food Agric 77, 213-222.

Huntington GB, Varga GA, Glenn BP \& Waldo DR (1988) Net absorption and oxygen consumption by Holstein steers fed alfalfa or orchardgrass silage at two equalized intakes. J Anim Sci 66, 1292-1302.

Huntington GB, Zetina EJ, Whitt JM \& Potts W (1996) Effects of dietary concentrate level on nutrient absorption, liver metabolism, and urea kinetics of beef steers fed isonitrogenous and isoenergetic diets. J Anim Sci 74, 908-916.

Institut National de Recherches Agronomiques (1978) Alimentation des Ruminants (The Feeding of Ruminants) [R Jarrige, editor].Versailles, France: INRA Publications.

Journet M, Huntington G \& Peyraud JL (1995) Le bilan des produits terminaux de la digestion (The assessment of the terminal products of digestion). In Nutrition des Ruminants Domestiques, Ingestion et Digestion, pp. 26-71 [R Jarrige, Y
Ruckebush, C Demarquilly, MH Farce and M Journet, editors]. Paris, France: INRA.

Katz ML \& Bergman EN (1969) A method for simultaneous cannulation of the major splanchnic blood vessels of the sheep. Am J Vet Res 30, 655-660.

Kelly JM, Park H, Summers M \& Milligan LP (1993) Interactions between protein and energy metabolism. In Quantitative Aspects of Ruminant Digestion and Metabolism, pp. 341-362 [JM Forbes and J France, editors]. Cambridge, MA: CAB International.

Kim H-S, Choung JJ, Chamberlain D \& Lobley GE (1999) Effect of propionate on ovine urea kinetics. In Proceedings of the VIIIth International Symposium on Protein Metabolism and Nutrition, p. 57 [GE Lobley, A White and JC MacRae, editors]. Wageningen, The Netherlands: Wageningen Press.

Koeln LL, Schlagheck TG \& Webb KE (1993) Amino acid flux across the gastrointestinal tract and liver of calves. J Dairy Sci 76, 2275-2285.

Kristensen NB, Pierzynowski SG \& Danfaer A (2000) Net portal appearance of volatile fatty acids in sheep intraruminally infused with mixtures of acetate, propionate, isobutyrate, butyrate and valerate. J Anim Sci 78, 1372-1379.

Lapierre H, Bernier JF, Dubreuil P, et al. (2000) The effect of feed intake level on splanchnic metabolism in growing beef steers. J Anim Sci 78, 1084-1099.

Lapierre H, Blouin JP, Lobley GE, Reynolds CK, Dubreuil P \& Bernier JF (1997) Effect of protein degradability on protein splanchnic metabolism in dairy cows. Proc Nutr Soc 56, 162A.

Lapierre H \& Lobley GE (2001) Nitrogen recycling in the ruminant: a review. J Dairy Sci 84, E223-E236.

Lien KA, Sauer WC \& Fenton M (1997) Mucin output in ileal digesta of pigs fed a protein free diet. Z Ernahrungswiss 36, $182-190$.

Lobley GE (1994) Amino acid and protein metabolism in the whole body and individual tissues of ruminant. In Principles of Protein Nutrition of Ruminants, pp. 147-178 [JM Asplund, editor]. London: CRC Press.

Lobley GE, Connell A, Revell DK, Bequette BJ, Brown DS \& Calder AG (1996b) Splanchnic-bed transfers of amino acids in sheep blood and plasma, as monitored through use of a multiple $\mathrm{U}-{ }^{13} \mathrm{C}$-labelled amino acid mixture. $\mathrm{Br} J$ Nutr 75, 217-235.

Lobley GE, Hoskin SO \& McNeil CJ (2001) Glutamine in animal science and production. J Nutr 131, 2525S-2531S.

Lobley GE, Milano GD \& Van der Walt JG (1999) The liver: integrator of nitrogen metabolism. IXth International Symposium on Ruminant Physiology, Pretoria, South Africa, pp. 149-168 [PJ Cronje, editor]. Wallingford, Oxon: CABI.

Lobley GE, Weijs PJM, Connell A, Calder AG, Brown DS \& Milne E (1996a) The fate of absorbed and exogenous ammonia as influenced by forage or forage-concentrate diets in growing sheep. Br J Nutr 76, 231-248.

MacRae JC, Cambell DR \& Eadie J (1975) Changes in biochemical composition of herbage upon freezing and thawing. J Agric Sci Camb 84, 125-131.

Majdoub L, Savary I, Bayle G, Grizard J, Vermorel M \& Ortigues-Marty I (1999) Répartition des nutriments azotés et des dépenses énergétiques entre le tube digestif, le foie et les muscles chez des agneaux recevant du ray-grass, stade pâturage (Distribution of nitrogenised nutrients and energy expenditure between the digestive tract, the liver and muscles in lambs receiving rye-grass, at the pasture stage). In Rencontres Recherches Ruminant, 6th ed., pp. 119-122.

Majdoub L, Vermorel M \& Ortigues-Marty I (2003) Intraruminal propionate supplementation modifies hindlimb energy metabolism without changing the splanchnic release of glucose in growing lambs. Br J Nutr 89, 39-50. 
Meijer AJ, Blommaart EFC, Dubbelhuis PF \& Van Sluijters DA (1999) Regulation of hepatic nitrogen metabolism. In Proceedings of the VIIIth International Symposium on Protein Metabolism and Nutrition, pp. 155-175 [GE Lobley, A White and JC MacRae, editors]. Wageningen, The Netherlands: Wageningen Press.

Milano GD, Holston-Moore A \& Lobley GE (2000) Influence of hepatic ammonia removal on ureagenesis, amino acid utilization and energy metabolism in the ovine liver. Br J Nutr 83, 307-315.

Moloney AP (1998) Growth and carcass composition in sheep offered isoenergetic rations which resulted in different concentrations of ruminal metabolites. Livest Prod Sci 56, 157-164.

Mukkur TK, Watson DL, Saini S \& Lascelles AK (1985) Purification and characterization of goblet-cell mucin of high Mr from the small intestine of sheep. Biochem J 229, 419-428.

Mutsvangwa T, Buchanan-Smith JG \& McBride BW (1997) Effects of ruminally degradable nitrogen intake and in vitro addition of ammonia and propionate on the metabolic fate of L- $\left[1-{ }^{14} \mathrm{C}\right]$ alanine and $\mathrm{L}-\left[{ }^{15} \mathrm{~N}\right] l y$ sine in isolated sheep hepatocytes. J Anim Sci 75, 1149-1159.

Newsholme EA \& Carrie AL (1994) Quantitative aspects of glucose and glutamine metabolism by intestinal cells. Gut 35, S13-S17.

Norton BW, Janes AN \& Armstrong DG (1982) The effects of intraruminal infusions of sodium bicarbonate, ammonium chloride and sodium butyrate on urea metabolism in sheep. Br J Nutr 48, 265-274.

Nozière P, Martin C, Rémond D, Kristensen NB, Bernard R \& Doreau M (2000) Effect of composition of ruminally-infused short-chain fatty acids on net fluxes of nutrients across portal-drained viscera in underfed ewes. Br J Nutr 83, 521-531.

Nozière P, Rémond D, Ferlay A \& Doreau M (1998) Differences between blood and plasma concentrations of acetate, $\beta$-hydroxybutyrate, glucose, ammonia and urea: implications for measurement of portal net fluxes in ewes. Reprod Nutr Dev 38, 509-518.

Obitsu T, Bremner D, Milne E \& Lobley GE (2000) Effect of abomasal glucose infusion on alanine metabolism and urea production in sheep. Br J Nutr 84, 157-163.

Ørskov ER, Meehan DE, MacLeod NA \& Kyle DJ (1999) Effect of glucose supply on fasting nitrogen excretion and effect of level and type of volatile fatty acid infusion on response to protein infusion in cattle. Br J Nutr 81, 389-393.

Ortigues I \& Doreau M (1995) Responses of the splanchnic tissues of ruminants to changes in intake: absorption of digestion end products, tissue mass, metabolic activity and implications to whole animal energy metabolism. Ann Zootech 44, 321-346.

Ortigues I \& Durand D (1995) Adaptation of energy metabolism to undernutrition in ewes. Contribution of portal-drained viscera, liver and hindquarters. Br J Nutr 73, 209-226.

Reeds PJ \& Burrin DG (2001) Glutamine and the bowel. J Nutr 131, 2505S-2508S.

Reeds PJ, Burrin DG, Stoll B \& Jahoor F (2000) Intestinal glutamate metabolism. J Nutr 130, 978S-982S.

Reeds PJ, Burrin DG, Stoll B \& van Goudoever JB (1999)
Consequences and regulation of gut metabolism. In Proceedings of the VIIIth International Symposium on Protein Metabolism and Nutrition, pp. 127-153 [GE Lobley, A White and JC MacRae, editors]. Wageningen, The Netherlands: Wageningen Press.

Rémond D, Bernard L \& Poncet C (2000a) Amino acid flux in ruminal and gastric veins and sheep, effects of ruminal and omasal injections of free amino acids and carnosine. J Anim Sci 78, 158-166.

Rémond D, Bernard L \& Poncet C (2000b) Free and peptide amino acid net flux across the rumen and the mesenteric- and portal-drained viscera of sheep. J Anim Sci 78, 1960-1972.

Rémond D, Meschy F \& Boivin R (1996) Metabolites, water and mineral exchanges across the rumen wall: mechanisms and regulation. Ann Zootech 45, 97-119.

Reynolds CK, Harmon DL, Prior RL \& Tyrrell HF (1994) Effects of mesenteric vein L-alanine infusion on liver metabolism of organic acids by beef heifers fed diets differing in forage:concentrate ratio. J Anim Sci 72, 3196-3206.

Reynolds CK, Tyrrell HF \& Reynolds PJ (1991) Effect of diet forage-to-concentrate ratio and intake on energy metabolism in growing beef steers: net nutrient metabolism by visceral tissues. J Nutr 121, 1004-1015.

Sakata T \& Tamate H (1978) Rumen epithelial cell proliferation accelerated by rapid increase in intraruminal butyrate. J Dairy Sci 61, 1109-1113.

Seal CJ \& Parker DS (1994) Effect of intraruminal propionic acid infusion on metabolism of mesenteric- and portal-drained viscera in growing steers fed a forage diet: I. Volatile fatty acids, glucose and lactate. J Anim Sci 72, 1325-1334.

Seal CJ \& Parker DS (1996) Effect of intraruminal propionic acid infusion on metabolism of mesenteric- and portal-drained viscera in growing steers fed a forage diet: II. Ammonia, urea, amino acids and peptides. J Anim Sci 74, 245-256.

Seal CJ, Parker DS, Balcells J \& Mole JL (1993) Nitrogen digestion in forage-fed sheep with and without intraruminal propionate infusion. J Agric Sci Camb 120, 107-114.

Stewart PM \& Walser M (1980) Failure of the normal ureagenic response to amino acids in organic acid-loaded rats. Proposed mechanism for the hyperammonemia of propionic and methylmalonic acidemia. $J$ Clin Invest 66, 484-492.

Storm E \& Ørskov ER (1983) The nutritive value of rumen micro-organisms in ruminants. Br J Nutr 50, 463-470.

Theurer CB, Huntington GB, Huber JT, Swingle RS \& Moore JA (2002) Net absorption and utilization of nitrogenous compounds across ruminal, intestinal, and hepatic tissues of growing beef steers fed dry-rolled or steam flaked sorghum grain. $J$ Anim Sci 80, 525-532.

Van der Walt JG (1993) Nitrogen metabolism in ruminant liver. Aust J Agric Res 44, 381-403.

Webb KE Jr (1999) Tissue, cellular and molecular aspects of peptide absorption and utilization. IXth International Symposium on Ruminant Physiology, Pretoria, South Africa, pp. 117-130 [PJ Cronje, editor]. Wallingford, Oxon: CABI.

Windmueller HG (1982) Glutamine utilization by the small intestine. Adv Enzymol Relat Areas Mol Biol 53, 201-237. 